\title{
Modelling of radial correlation reflectometry in cylinder geometry.
}

\author{
Gusakov E.Z. ${ }^{1}$, Bulanin V.V. ${ }^{2}$, Rozhdestvenskiy O.I. ${ }^{2}$ \\ Ioffe Institute, Politekhnicheskaya 26, 194021 St.Petersburg, Russia ${ }^{1}$ \\ *SPbPU Politekhnicheskaya 29, 195251 St.Petersburg, Russia ${ }^{2}$ \\ evgeniy.gusakov@mail.ioffe.ru
}

\section{Introduction.}

Fluctuation reflectometry is a widely used technique providing information on the tokamak plasma small scale turbulence. Technical simplicity and operation at a single access to plasma are among its merits, which however cause interpretation problems related to localization of measurements and wave number resolution. In order to improve the fluctuation reflectometry wave number selectivity a more sophisticated radial correlation reflectometry (RCR), using simultaneously different frequencies for probing was proposed. The coherence decay of two scattering signals with growing difference of probing frequencies is studied in this diagnostic and applied for estimation of the turbulence radial correlation length. This estimation is often performed in a manner similar to that developed in the probe correlative technique, namely it is supposed that fluctuating part of the reflectometer signal is produced by backscattering in the nearest vicinity of the cut off point so that the coherence dependence on probing frequency difference can be interpreted as dependence on the separation of measurement points.

However already in 1D numerical Born approximation analysis [1] a role of small angle scattering was shown, reducing the diagnostic spatial resolution and leading to a slow decay of coherence in RCR. This effect was confirmed in RCR linear analytic theory in 1D and slab 2D model [2-4]. It was also resolved in1D full wave numerical modeling [5] at small level of density perturbations and sufficiently well modeled long scale component of the turbulence wave number spectrum.

In the present paper the RCR CCF is analyzed in linear, Born approximation but, unlike [1-5], in cylinder geometry, which allows to describe the magnetic surface curvature effects.

\section{The theoretical model.}

We analyze the $\mathrm{O}$ mode RCR using the 2D cylinder geometry model in which the probing and scattered wave electric field is parallel to the magnetic field and is described by the following equation

$$
\frac{\partial^{2} E}{\partial r^{2}}+\frac{1}{r} \frac{\partial E}{\partial r}+\frac{1}{r^{2}} \frac{\partial^{2} E}{\partial \vartheta^{2}}+\frac{1}{c^{2}}\left\{\omega_{i}^{2}-\frac{4 \pi e^{2}[n(r)+\delta n(r, \vartheta)]}{m_{e}}\right\} E=0
$$

where $\omega_{i}$ stands for the variable probing frequency in the RCR signal channel (whereas $\omega_{0}$ stands for the frequency in the reference channel); $n(r)$ is the density profile and $\delta n(r, \vartheta)$ is a random density perturbation which is given by a superposition of harmonics

$$
\delta n^{i}(r, \theta)=\frac{n(r)}{n_{0}} \sum_{k, l} \delta n_{k l} \cos \left(\kappa_{k} r+q_{l} r \theta+\varphi_{k l}^{i}\right)
$$

possessing wave numbers $\kappa_{k}=\frac{k}{L} ; q_{l}=\frac{l}{r}$ and random phase $\varphi_{k l}^{i}$. The length $L$, determining the maximal fluctuation radial wave length $(2 \pi L)$ in majority of computations was taken $L=135$ $\mathrm{cm}$. 
The density perturbation level is supposed to be proportional to the local density, whereas the fluctuation spectrum $\delta n_{k l}^{2}$ is assumed Gaussian given by the following expression: $\delta n_{k l}^{2}=\exp \left(-\left(\frac{k l_{c}}{L}\right)^{2}\right) \exp \left(-\left(\frac{l l_{c}}{r}\right)^{2}\right)$. The RCR performance modeling is performed for three types of the density profile ("linear", "convex" and "concave") shown in figure 1. The probing frequency in the reference channel was taken equal to $63.1 \mathrm{GHz}$
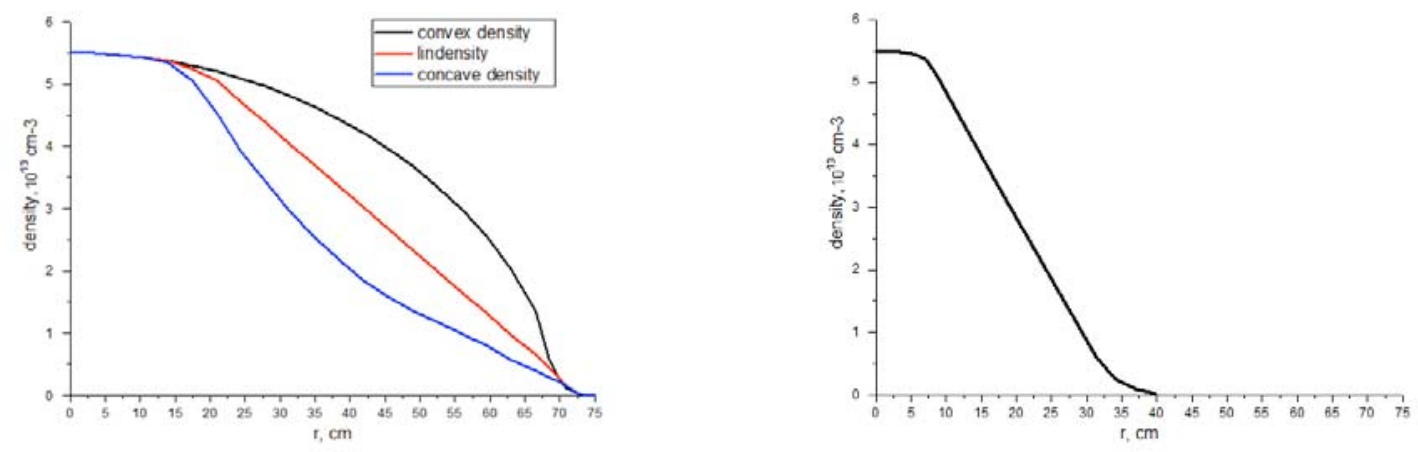

Figure 1. Density profiles. (Convex, linear and concave for "large" plasma (a) and linear for "small" plasma (b))

In the case of low density perturbation level satisfying condition $\quad \frac{\omega^{2} l_{c} x_{c}}{c^{2}}\left(\frac{\delta n}{n_{c}}\right)^{2} \ln \frac{x_{c}}{l_{c}}<<1$ (here $x_{c}$ stands for the local density gradient scale length in the cut off, where the plasma density is equal to $n_{c}$ ), according to [2], the Born approximation linear in fluctuation amplitude is valid. The most convenient expression for the reflectometry signal picked up by the ideal single mode antenna in this approximation is provided by the reciprocity theorem [6]

$$
A_{s}\left(\omega_{j}\right)=\frac{i \omega_{j} \sqrt{P_{i}}}{16 \pi} \int \frac{\delta n(r, \vartheta)}{n_{c}} E^{2}\left(\omega_{j}, r, \vartheta\right) r d r d \vartheta,
$$

where $E^{2}\left(\omega_{j}, r, \vartheta\right)$ gives the unite power probing wave electric field distribution, $P_{i}$ and $\left|A_{s}\left(\omega_{j}\right)\right|^{2}=p_{s}\left(\omega_{j}\right)$ are correspondingly the probing and scattered power. The probing wave electric field distribution in the plasma volume is computed for $\delta n(r, \vartheta)=0$ using equation (1) under assumption that at the plasma boundary it takes a form of Gaussian beam which is characterized by half width $\rho$ varying from $1.5 \mathrm{~cm}$ to $8 \mathrm{~cm}$ and plane phase front.

\section{The RCR cross-correlation function computations.}

The RCR cross-correlation function $(\mathrm{CCF})$ given by the following expression:

$$
C C F_{12}=\frac{\left\langle A_{s}\left(\omega_{1}\right) A_{s}^{*}\left(\omega_{2}\right)\right\rangle}{\sqrt{p_{s}\left(\omega_{1}\right) p_{s}\left(\omega_{2}\right)}}
$$

was computed in the cylinder geometry for different density profiles, antennae diagrams and turbulence correlation lengths. The dependence of the CCF absolute value (coherence) on the channel frequency difference is demonstrated in Figure $2 \mathrm{a}$ for the three density profiles shown in Figure 1. In spite of the fact the dependencies are different, all of them possess pronounced slow decaying tails. It is important to note that when plotted against the cut off separation, as in Figure $2 \mathrm{~b}$ all the dependencies practically coincide, being however substantially different from the density fluctuations correlation function, shown by black solid line in Figure $2 b$. 

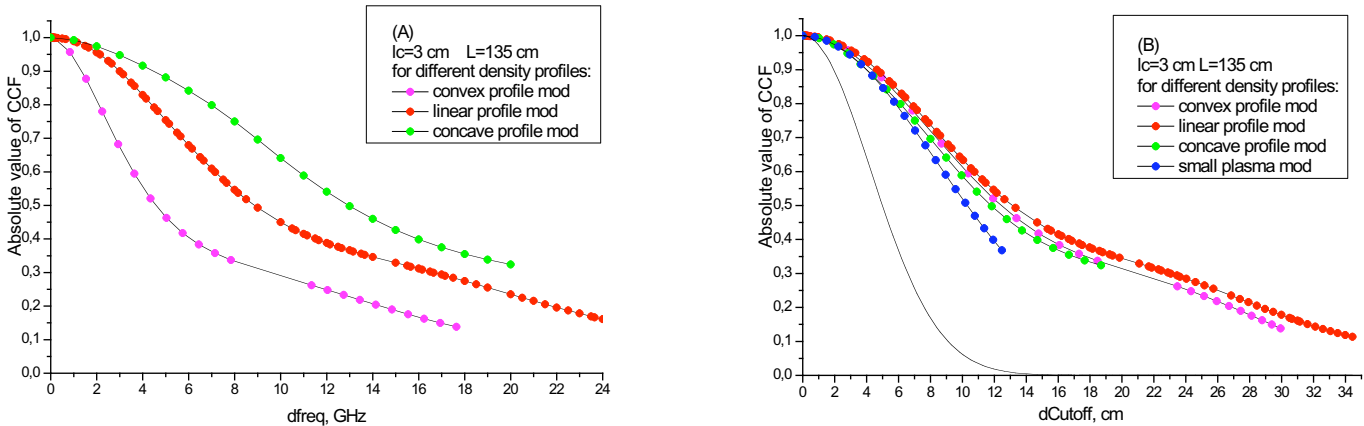

Figure 2. RCR CCF for different profiles. The coherency is plotted against the frequency difference (a) and cut offs separation (b).

The shape of this universal dependence is practically not sensitive to the antenna beam width variation leading to the transition of the cut off position from the near to the wave zone of the antenna (See Figure 3). It is also only weakly sensitive to the choice of the minimal fluctuation wave number until it is smaller than the inverse distance between the cut off and the plasma boundary (See Figure 4). It should be mentioned that the suppression of the slowly decaying CCF tail associated with suppression of long scales in the fluctuation spectrum was shown recently in $1 \mathrm{D}$ numerical modeling [5].

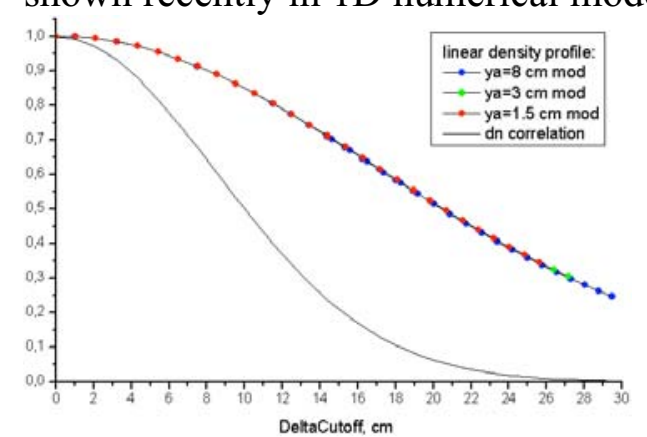

Figure 3. RCR CCF for different beam

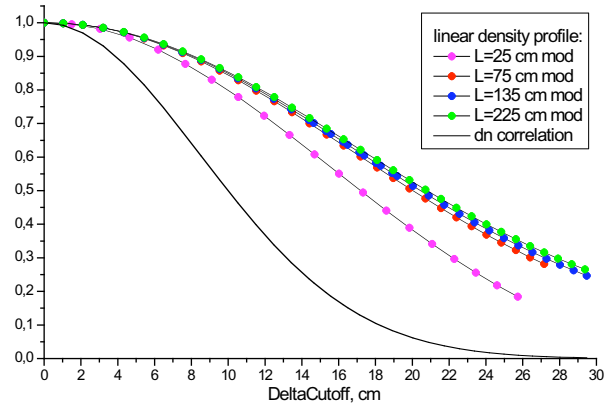

Figure 4. RCR CCF for different minimal fluctuation wavelength

The influence of the turbulence correlation length on the CCF form is shown in Figure $5(a, b)$, where the CCF is plotted against the frequency difference in channels (a) and the cut offs separation normalized to the turbulence correlation length (b). As it is seen in Figure 5b, the slowly decaying CCF tail is decreased with the growing turbulence correlation length in agreement with predictions of analytical 2D slab geometry theory [3]. Nevertheless even for the correlation length comparable to the distance from cut off to the plasma boundary, the RCR CCF is significantly different from that of the turbulence.

a)

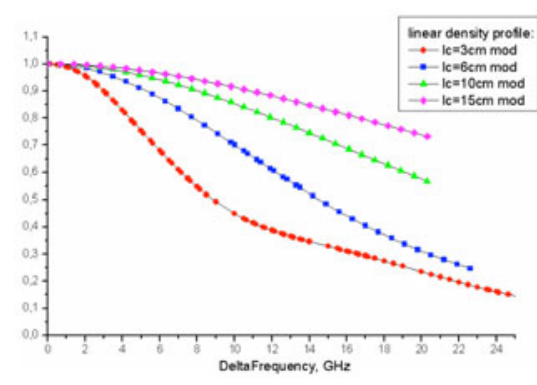

b)

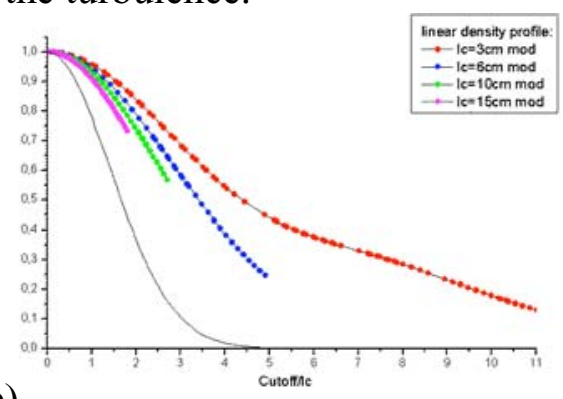

Figure 5. The RCR CCF for different turbulence correlation length versus probing frequency difference (a) and normalized cut off separation (b). 


\section{Discussion.}

The CCF dependencies obtained numerically in the previous section for cylinder experiment geometry possess features similar to those demonstrated analytically in 1D and 2D slab model. Universal dependence on normalized cut offs separation, different from that of the turbulence CCF, and slow coherency decay are among these essential features. These universality and difference are explained in analytics based on the WKB approximation $[2,4]$ by enhanced contribution of long radial scales to the reflectometry signal.

Strictly speaking the model developed in $[2,4]$ is only applicable to long scale turbulence possessing the correlation length exceeding the so called Airy scale (providing the size of the probing wave electric field lobe at the cut off) $l_{c}>\left(\frac{c^{2} x_{c}}{\omega^{2}}\right)^{1 / 3}$. Nevertheless, on one hand this condition is satisfied in majority of reflectometry experiments and on the other hand the 2D theory developed in [3] in slab plasma model for linear density profile, but arbitrary turbulence scales, also confirms the conclusion on the enhanced contribution of long scales into the RCR signal and, as a consequence, into the CCF.

\section{Conclusions.}

Summarizing the paper results we would like to stress that a $2 \mathrm{D}$ numerical study of the RCR performed in cylinder geometry have shown that the RCR CCF is a universal function of the cut off separation only weakly sensitive to the details of reflectometry experiment and plasma parameters, however different from the turbulence CCF. This difference is explained by enhancement of reflectometry scattering efficiency at small radial wave numbers which was demonstrated in simple 1D WKB and 2D slab geometry models. Thus the CCF in the form it is provided by the RCR is not suitable for direct determination of the turbulence correlation length. Development of the spectrum reconstruction procedure based on the CCF dependence on cut offs separation needed for this purpose is in progress at present.

\section{Acknowledgements.}

Financial support of RFBR grants 06-02-16785, 06-02-17212, 07-02-92162-CNRS, NWORFBR grant 047.016.015 and INTAS grant 05-8046 is acknowledged.

1. Hutchinson I 1992 Plasma Phys. Control. Fusion 341225

2. Gusakov E Z and Popov A Yu 2002 Plasma Phys. Control. Fusion 442327

3. Gusakov E Z and Yakovlev B O 2002 Plasma Phys. Control. Fusion 442525

4. Gusakov E Z and Popov A Yu 2004 Plasma Phys. Control. Fusion 461393

5. G Leclert, S Heuraux, E Gusakov et al. 2006 Plasma Phys. Control. Fusion 481389

6. Piliya A D and Popov A Yu 2002 Plasma Phys. Control. Fusion 44467 